\section{Military Technical College Kobry El-kobbah, Cairo, Egypt}

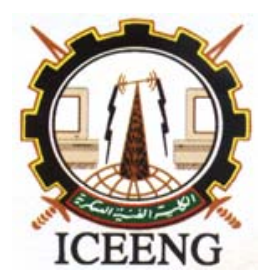

\section{$5^{\text {th }}$ International Conference on Electrical Engineering ICEENG 2006}

\title{
FAULT DETECTION OF THE DATA DISPLAYING SYSTEM IN MARINE CENTERS
}

\author{
Emad* M. Hamad, Mohamed** A. Abd El-Rahman, Mohamed*** E. Nasr \\ and Usama**** B. Sabbah
}

\begin{abstract}
This paper presents a comprehensive analysis of the vessel traffic management system (VTMS). It is shown that displaying system includes the signal state board, with its four driving boards, and station PC. Each driving board feeds one row. We show that, as an example, Row 2 contains six $(7 \times 5$ dot matrix $)$ modules and each dot has its own flipping coil. In addition, each driving board has 58 Darlington transistors. The address and data lines of the processor Z80180 are used to control the flipping circuit through decoders, addressable latches, buffers and inverters. Interrupt circuit generates signal $\overline{I N T 1}$ which is used by the processor to switch off all transistors after each dot flipping process. We have built an assembly EPROM program to deal with the two assembly subroutines, activated by $\overline{I N T} 1$ and $\overline{I N T 2}$ interrupt signals, to detect faults associated with the flipping circuit transistors. We performed a new visual basic program(paper under publication) in the station PC to handle the detected faults by EPROM program.This work is illustrated practically by tracing measured signals at different points for the example of short and open transistor.
\end{abstract}

\section{Introduction}

The subject of the paper is to analyze the displaying data and detect faults of the flipping circuit components in the system applied in Suez Canal. The main objective of the VTMS resides on minimizing accidents to ensure safe passing of the vessels. In this paper, suggested procedures are implemented to determine the faulty transistor or the digital electronic components connected with it.

\section{Interrupt and Flipping Circuits Analysis}

In the flipping circuit shown in Fig.1, the current passes through T46-D1-coil-T3-R22 when T46, T3 are switched ON and T32 and T17 are switched OFF. The current passes in the opposite direction when T32 and T17 are switched ON and T46, T3 are switched OFF. The

\footnotetext{
* Post graduate student, Suez Canal Authority, Egypt.

** Dr., Air Defense College, Alexandria, Egypt.

*** Professor, Electronics and Communication Department, Tanta University, Egypt.

**** Dr., Director of Planning and Researches Department, Suez Canal Authority, Egypt.
} 
dot will turn to the yellow face in the first case and to black face in the second one. Fig. 3 depicts that the current passing through the inductor, rising as a ramp, causes a voltage drop across the resistor R 22 of value $1 \Omega$. This voltage is normally $4.8 \mathrm{~V}$ with $380 \mu \mathrm{sec}$ duration.

The interrupt circuit shown in Fig. 2 includes two identical wave shaping circuits which are simply RC circuit, two comparators COMP1, COMP2 and two XOR gates. $\mathrm{R}_{3}-\mathrm{C}_{10}$ and $\mathrm{R}_{5}-\mathrm{C}_{12}$ represent these two shaping circuits respectively.

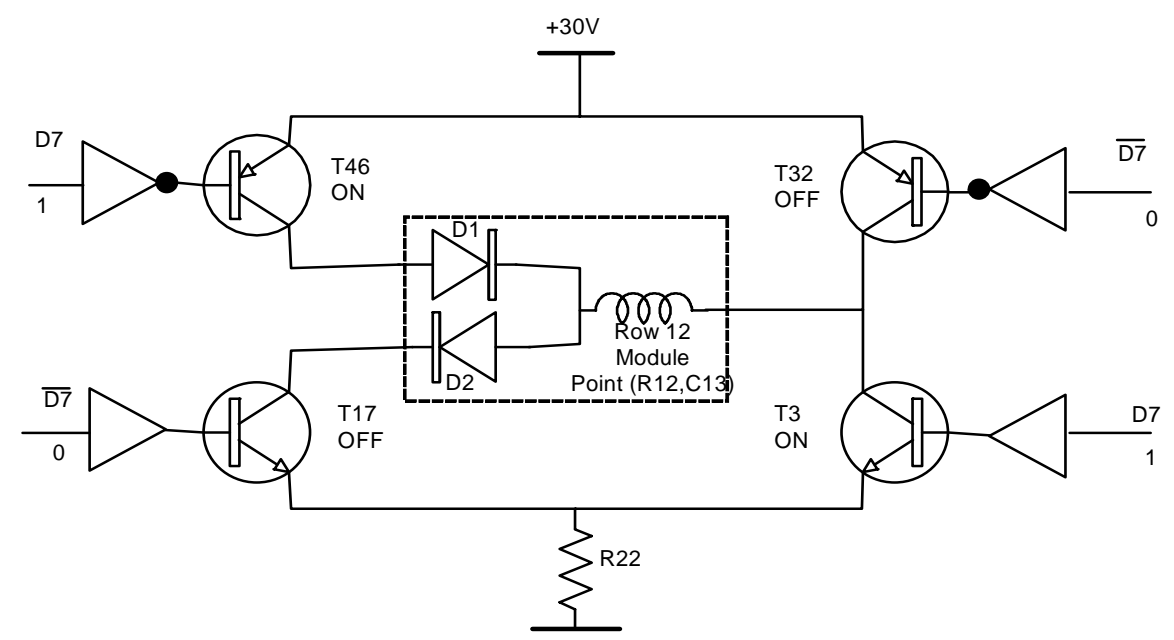

Fig.1. A simplified circuit for flipping single point of (DotMatrix/7 Segments) module

The time constant of these circuits is:

$\tau=R_{3} C_{10}=R_{5} C_{12}=100 \mathrm{k} \Omega \times 2.2 n F=220 \mu \mathrm{sec}$.

Therefore the capacitor $\mathrm{C}_{10}$ will charge according to the following the equation:

$V_{o}=V_{\text {inp }}\left(1-e^{-t / \tau}\right)$

Where $V_{\text {inp }}=4.8 \mathrm{~V}$. The output voltage of the shaping circuit across the capacitors $C_{10}$ and $C_{12}$ after $t=380 \mu \mathrm{sec}$ becomes $3.9 \mathrm{~V}$. These capacitors will decay to approximately zero voltage value after $t=5 \tau$ according to $e^{-t / \tau}$ as shown in Fig. 4 . This voltage is fed to the inverting terminals of the two comparators COMP1 and COMP2. The reference voltage at the non-inverting terminals of COMP1, COMP2 is $3.4 \mathrm{~V}$ and $2 \mathrm{~V}$ respectively. The output waveforms of COMP1 and COMP2 are illustrated in Fig.5 and Fig.6. The output of COMP1 is fed to the pin (9) of the XOR1 and its pin (10) is connected to pin (10) of addressable latch IC21 (74HC259). This allows the processor Z80180 to control the output of the XOR1 which is connected to the $\overline{I N T 1}$ pin. Similarly, the output of COMP2 is fed to the pin (13) of the XOR2 and its pin (12) is connected to pin (11) of addressable latch IC21 (74HC259). This allows the processor Z80180 to control the output of the XOR2 which is connected to the $\overline{I N T 2}$ pin.

In the EPROM program of the driving board, the initial settings are as follow:

i) INT/TRAP control register "ITC" with the address $(34 \mathrm{H})$ enables the external interrupts $\overline{I N T 1}, \overline{I N T 2}$ and disables the external interrupt $\overline{I N T 0}$. This is done in subroutine (09DEH) which has the following instructions:

09E8 LD A, (06H)

09EA OUT0 (34H), A 


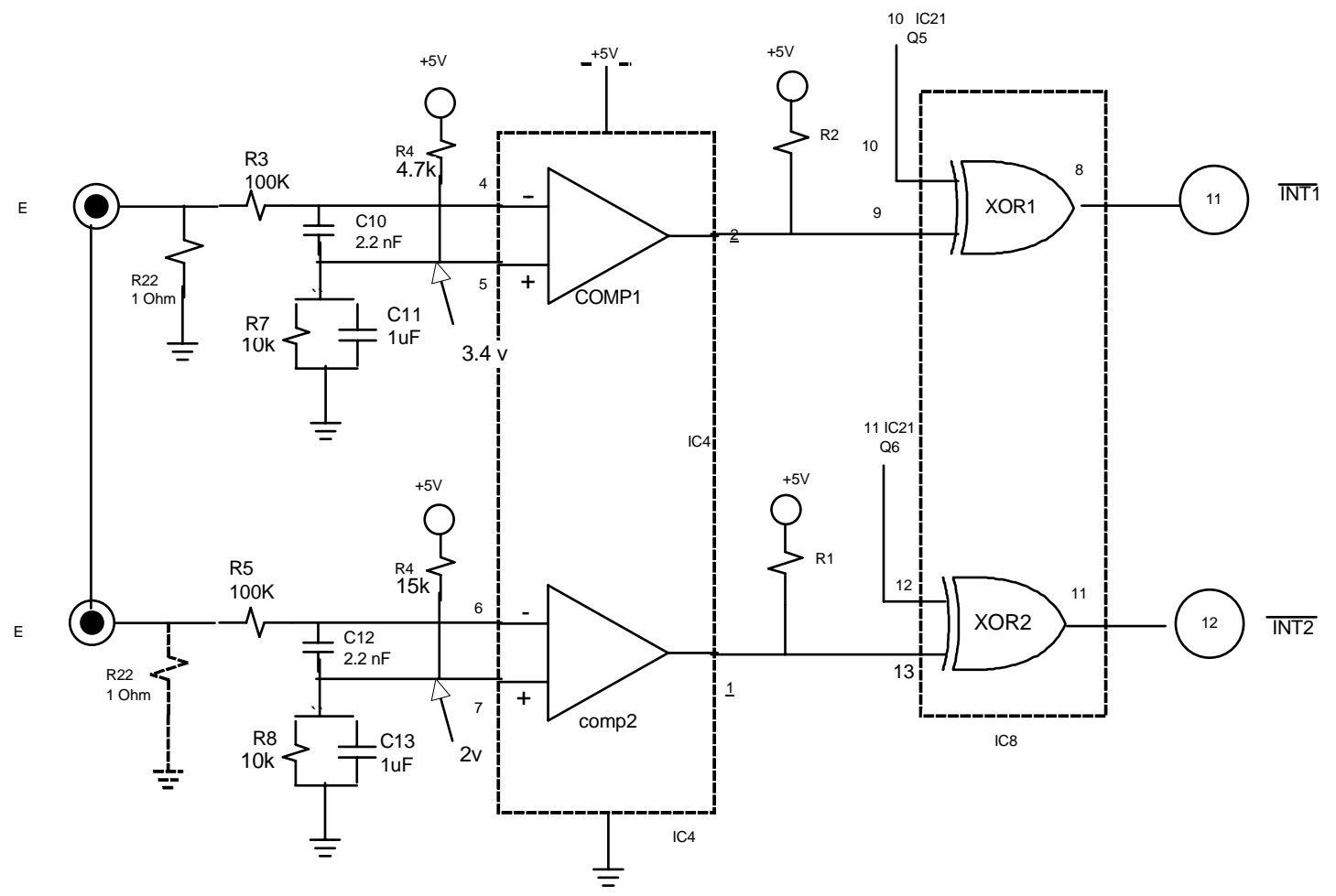

Fig.2. Interrupt Interface circuit

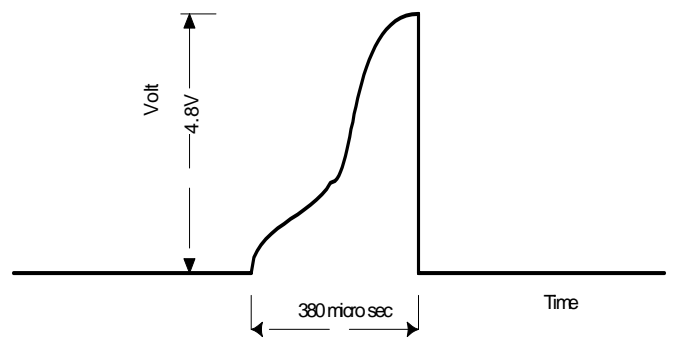

Fig.3. Voltage across R22 " point E and Gnd " Pin \#2 IC4 (o/p of comp1)

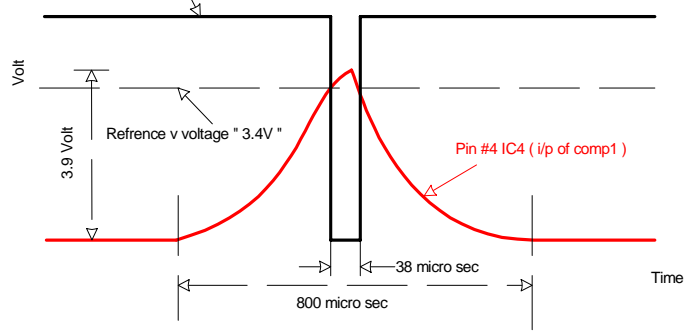

Fig.5. Pins "4" ," 2" IC4 comparator

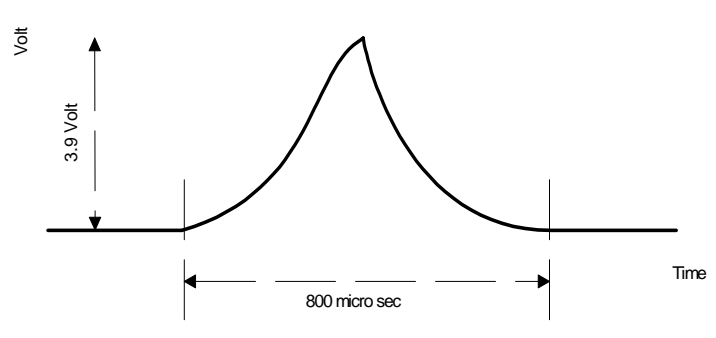

Fig.4. Pins "4", " 6" IC4 comparator Pin \#1 IC4 (o/p of comp2)

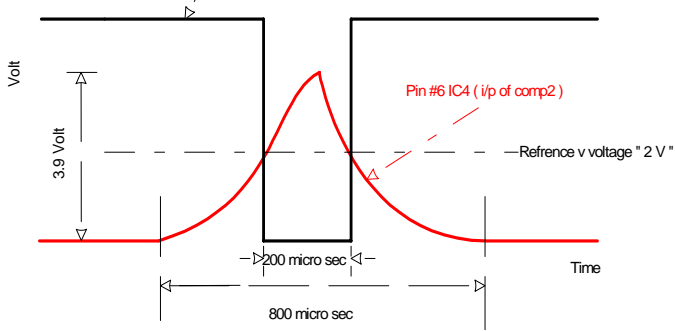

Fig.6. Pins "4", "6" IC4 comparator 


\begin{tabular}{|c|c|c|c|c|c|c|c|}
\hline \hline TRAP & UF0 & & & & ITE2 & ITE1 & ITE0 \\
\hline \hline R/W & R & & & & R/W & R/W & R/W \\
\hline \hline 0 & 0 & 0 & 0 & 0 & 1 & 1 & 0 \\
\hline \hline
\end{tabular}

Enable external interrupt 2

ii) Initial adjustment for memory location $(9291 \mathrm{H})$ and XOR1 gate:
09ED
LD A, $00 \mathrm{H}$
09EF
LD $(9291 \mathrm{H}), \mathrm{A}$
09F2
OR $(15 \mathrm{H})$
09F4
OUT (0FH), A

These instructions store $(00 \mathrm{H})$ in the memory location $(9291 \mathrm{H})$ which is used as a flag to the $\overline{I N T} 1$ subroutine, then OUT $(15 \mathrm{H})$ at the address $(0 \mathrm{FH})$. This leads to

Data lines

\begin{tabular}{|c|c|c|c|c|c|c|c|}
\hline $\mathrm{D}_{7}$ & $\mathrm{D}_{6}$ & $\mathrm{D}_{5}$ & $\mathrm{D}_{4}$ & $\mathrm{D}_{3}$ & $\mathrm{D}_{2}$ & $\overline{\mathrm{D}_{1}}$ & $\mathrm{D}_{0}$ \\
\hline 0 & 0 & 0 & 1 & 0 & 1 & 0 & 1 \\
\hline \multicolumn{8}{|c|}{ Address llines } \\
\hline $\mathrm{A}_{7}$ & $\mathrm{~A}_{6}$ & $\mathrm{~A}_{5}$ & $\mathrm{~A}_{4}$ & $\mathrm{~A}_{3}$ & $\mathrm{~A}_{2}$ & $\mathrm{~A}_{1}$ & $\mathrm{~A}_{0}$ \\
\hline 0 & 0 & 0 & $\overline{0}$ & 1 & 1 & 1 & 1 \\
\hline
\end{tabular}

The address lines A3- A5 are 001 which are connected to the IC11 3-8 decoder (74HC138) causing IC12 and IC14 to be enabled. The address lines A0-A2 are 111, this will select Q7 in IC12-IC14 (74HC259) which nothing connected to it as shown in Fig 7.

The data lines D3- D5 are 010 which are connected to 3-8 decoder (74HC138) to enable IC21. D0- D2 [101] are input to addressable latch IC21 (74HC259) causing D7 (logic 0) to pass through the output Q5. This output (logic 0 ) is fed to XOR1 pin \#10, and XOR1 will work as a buffer connecting the output of COMP1, shown in Figs. 3, to $\overline{I N T 1}$ pin of the processor.

iii) Initial adjustment for memory location $(9292 \mathrm{H})$ and XOR2 gate:

09F6 LD A, (00H)

09F8 LD $(9292 \mathrm{H}), \mathrm{A}$

09FB OR (16H)

09FD OUT (0FH)

These instructions store $(00 \mathrm{H})$ in the memory location $(9292 \mathrm{H})$ which is used as a flag to $\overline{I N T 2}$ subroutine. Then out the byte $(16 \mathrm{H})$ at the address $(0 \mathrm{FH})$.

Data lines

\begin{tabular}{|c|c||c||c||c||c||c||c||}
\hline \hline D7 & D6 & D5 & D4 & D3 & D2 & D1 & D0 \\
\hline \hline 0 & 0 & 0 & 1 & 0 & 1 & 1 & 0 \\
\hline \hline
\end{tabular}

Address lines

\begin{tabular}{|c|c|c|c|c|c|c|c|}
\hline $\mathrm{A}_{7}$ & $\mathrm{~A}_{6}$ & $\mathrm{~A}_{5}$ & $\mathrm{~A}_{4}$ & $\mathrm{~A}_{3}$ & $\mathrm{~A}_{2}$ & $\mathrm{~A}_{1}$ & $\mathrm{~A}_{0}$ \\
\hline \hline 0 & 0 & 0 & 0 & 1 & 1 & 1 & 1 \\
\hline
\end{tabular}




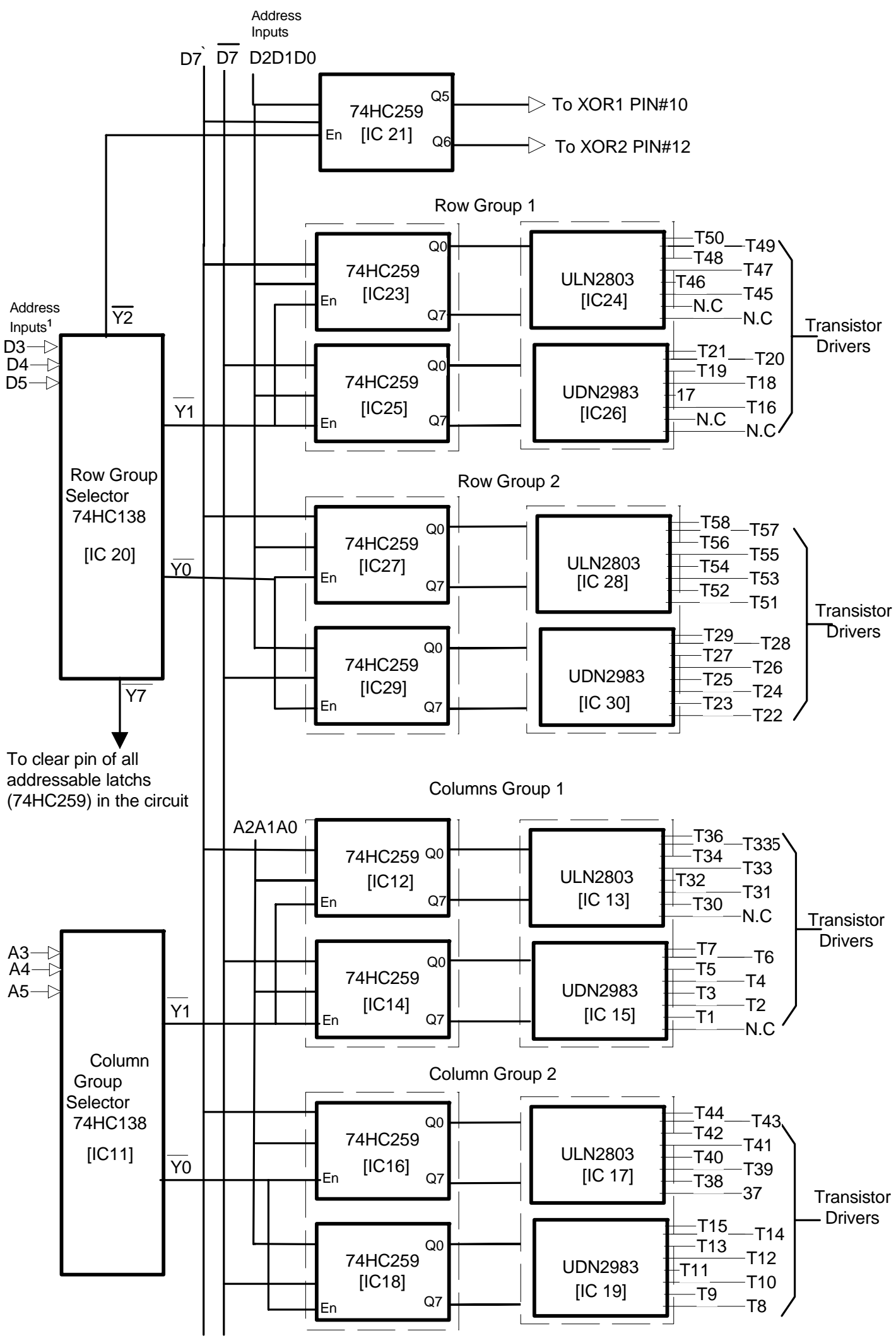

Fig.7. Rows and Columns selector 
As mentioned before, IC12 \& IC14 are enabled, which in conjunction with A0-A2 (111) leads to select nothing as shown in Fig 7 ..

The data lines D3- D5 are 010 which are connected to 3-8 decoder (74HC138) to enable IC21. D0- D2 [110] are input to addressable latch IC21 (74HC259) causing D7 (logic 0) to pass through the output Q6. This output (logic 0) is fed to XOR2 pin (12), and XOR2 will work as a buffer connecting the output of COMP 2, shown in Fig.3, to $\overline{I N T 2}$ pin of the processor.

In the proceeding section, we will continue to explain the normal operation for the flipping process of any dot. When the current passing through the coil, the voltage drop across R22 is shaped and compared with the reference values in COMP1 and COMP2. This leads to the output waveforms in Figs. 5,6 to be appeared at the inputs of XOR1 and XOR2 respectively. We note that the duration of interrupt signal $\overline{I N T 1}$ (logic 0 ) is $38 \mu \mathrm{sec}$. Also the duration of interrupt signal $\overline{I N T 2}(\operatorname{logic} 0)$ is $200 \mu \mathrm{sec}$. This shows that the $\overline{I N T 2}$ occurred first and the processor Z80180 interrupted by $\overline{I N T 2}$ at location (0BFFH) and begins to execute side A of the flow chart in Fig. 8 . It stores logic 1 at bits 1,4 at memory location $(9290 \mathrm{H}),(80 \mathrm{H})$ at memory location $(9292 \mathrm{H})$ to make the program follows side $\mathrm{B}$ at the next execution of interrupt 2 subroutine. Then the processor will output $(96 \mathrm{H})$ at the address $(0 \mathrm{FH})$ causing address lines to select Q7 at IC12 and IC14 which is not connected. The data lines are:

\begin{tabular}{|c|c|c|c|c|c|c|c|}
\hline D7 & D6 & D5 & D4 & D3 & D2 & D1 & D0 \\
\hline 1 & 0 & 0 & 1 & 0 & 1 & 1 & 0 \\
\hline $\begin{array}{l}\text { Output on } \\
\text { Q6 } \\
(47 \mathrm{HC} 259)\end{array}$ & & \multicolumn{2}{|c|}{$\begin{array}{l}\text { Select } \\
(74 \mathrm{HC} 259)\end{array}$} & IC21 & Selec & $\mathrm{in}$ & \\
\hline
\end{tabular}

The input to XOR2 pin (13) is logic 1 . Therefore the XOR2 works as an inverter it will invert the low coming from the comp2 to logic 1 which is applied to INT2 pin (12) of the processor Z80180 as shown in Fig.3. After a short time the processor will have low at pin (11) $\overline{I N T 1}$. Therefore the processor will be interrupted.

The processor starts executing side A of the flow chart related to $\overline{I N T} 1$ subroutine as shown chart in Fig.9. The first instruction causes the data lines to be as follows

\begin{tabular}{|c||c|c|c||c||c|c||c|}
\hline D7 & D6 & D5 & D4 & D3 & D2 & D1 & D0 \\
\hline \hline 0 & 0 & 1 & 1 & 1 & 0 & 0 & 1 \\
\hline
\end{tabular}

Not

effected clears all the addressable Not effected latches 


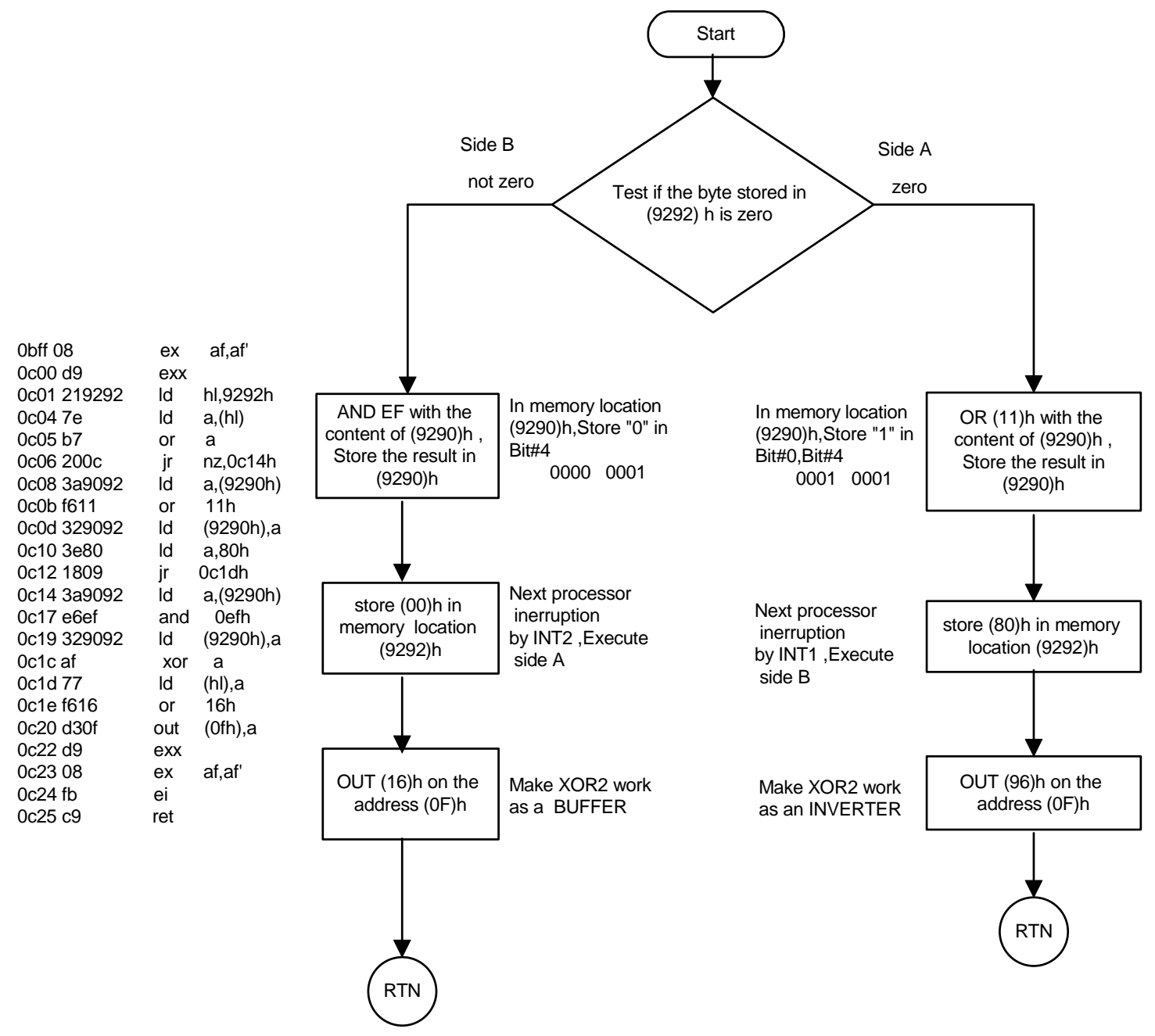

Fig.8. Interrupt 2 subroutine flow chart

The processor switches OFF all transistors on the board. It stores logic 1 at bits 2,5 at memory location $(9290 \mathrm{H}),(80 \mathrm{H})$ at memory location $(9291 \mathrm{H})$ to make the program follow side $\mathrm{B}$ at the next execution of interrupt 1 subroutine. Then the processor will out $(95 \mathrm{H})$ at the address $(0 \mathrm{FH})$ causing address lines to select Q7 at IC12 and IC14 which is not connected. The data lines are:

\begin{tabular}{|c|c|c|c|c|c|c|c|}
\hline D7 & D6 & D5 & D4 & D3 & $\mathrm{D} 2$ & D1 & D0 \\
\hline 1 & 0 & 0 & 1 & 0 & 1 & 0 & 1 \\
\hline
\end{tabular}




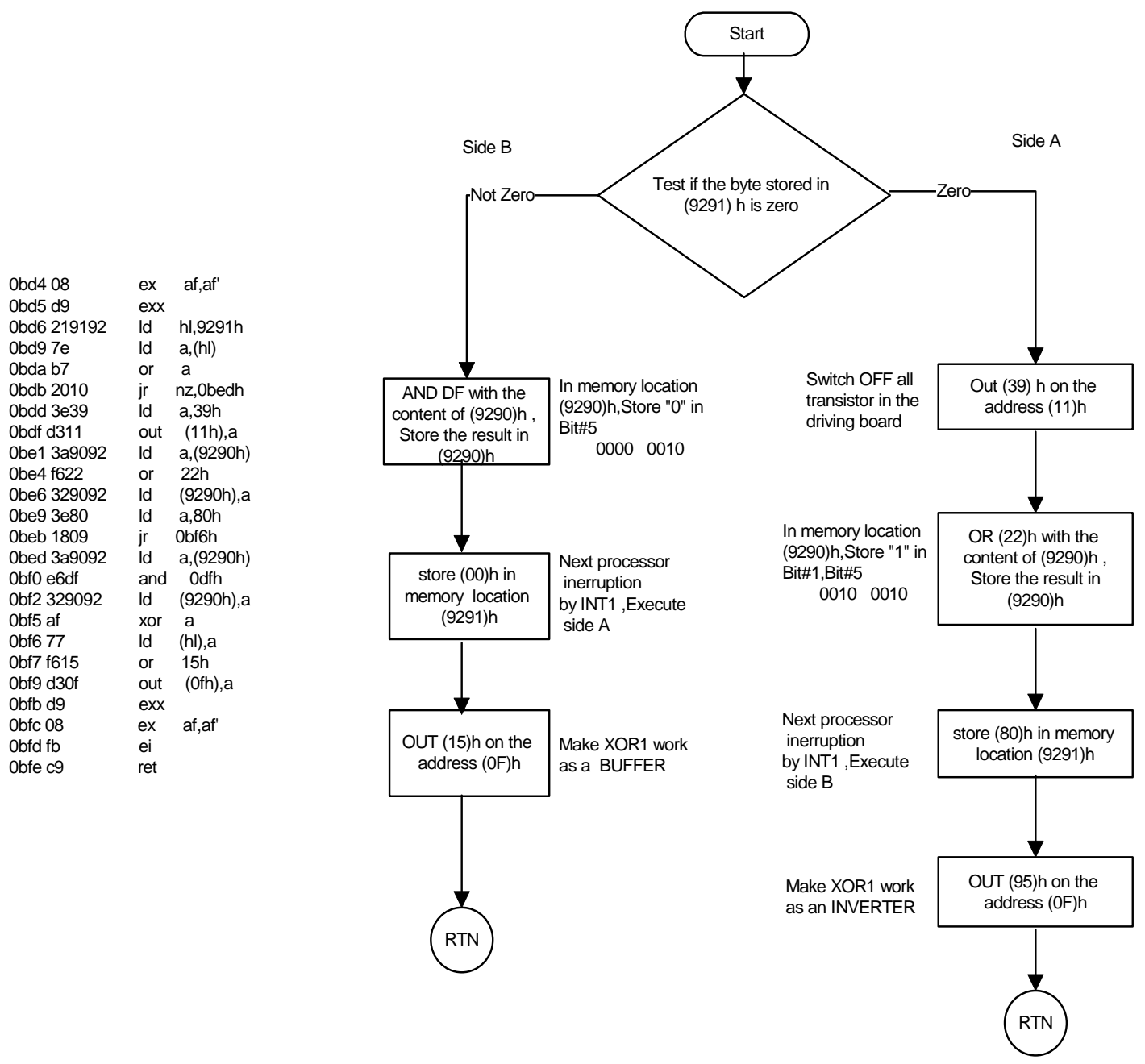

Fig.9. Interrupt 1 subroutine flow chart

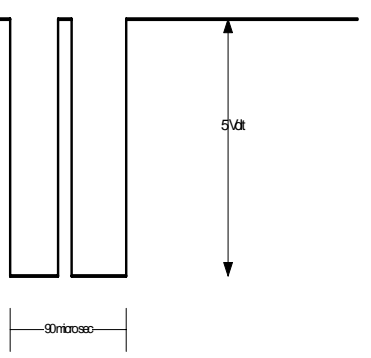

Fig.11. Pin "8" IC8 to processor INT1

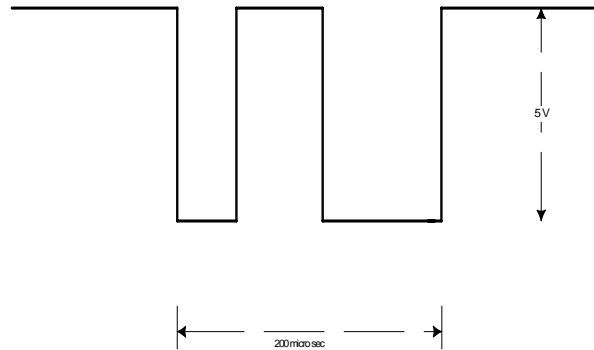

Fig.10. Pin "11" IC8 to processor INT2

The input to XOR1 pin (10) is logic 1 . Therefore the XOR1 works as an inverter it will invert the low coming from the comp1 to logic 1 which is applied to INT1 pin (11) of the processor Z80180 as shown in Fig.3.

As shown in fig.5. the output of the comp1 is logic 0 for about $38 \mu \mathrm{Sec}$ before returning to logic 1 . Therefore after the low period $(38 \mu \mathrm{Sec})$ the output of XOR1, which acts as an 
inverter, will be low again as shown in Fig.10. Thus the processor will interrupted again by $\overline{I N T 1}$ executing side $\mathrm{B}$ of the $\overline{I N T 1}$ subroutine.

It stores logic 0 at bits 5 at memory location $(9290 \mathrm{H}),(00 \mathrm{H})$ at memory location $(9291 \mathrm{H})$ to make the program follows side $\mathrm{A}$ at the next execution of interrupt 1 subroutine. Then the processor will out $(15 \mathrm{H})$ at the address $(0 \mathrm{FH})$ causing address lines to select Q7 at IC12 and IC14 which nothing connected to it as shown in Fig.7. The data lines are:

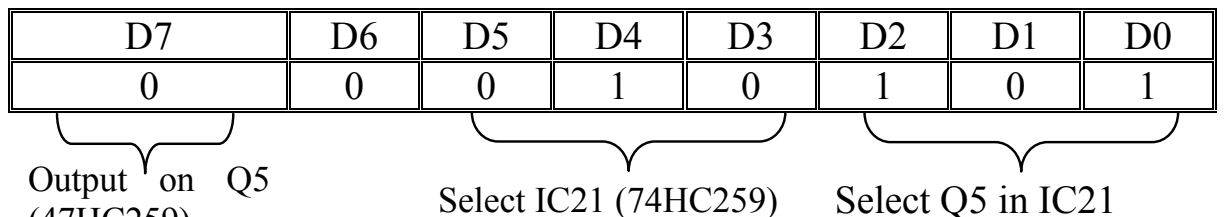

(47HC259)

The input to XOR1 pin (10) is logic 0. Therefore the XOR1 works as a buffer, it will apply the high coming from the comp1 to INT1 pin (11) of the processor Z80180.

As shown in figure (6) the output of the comp2 is logic 0 for about $200 \mu \mathrm{Sec}$ before returning to logic 1 . Therefore after the low period $(200 \mu \mathrm{Sec})$ the output of XOR2, which acts as an inverter, will be low again as shown in Fig.11. Thus the processor will interrupted again by $\overline{I N T 2}$ executing side $\mathrm{B}$ of the $\overline{I N T 2}$ subroutine.

It stores logic 0 at bits 4 at memory location $(9290 \mathrm{H}),(00 \mathrm{H})$ at memory location $(9292 \mathrm{H})$ to make the program follows side $A$ at the next execution of interrupt 1 subroutine. Then the processor will out $(16 \mathrm{H})$ at the address $(0 \mathrm{FH})$ causing address lines to select Q7 at IC12 and IC14 which nothing connected to it as shown in Fig.7. The data lines are:

\begin{tabular}{|c|c|c|c|c|c|c|c|}
\hline D7 & D6 & D5 & D4 & D3 & D2 & D1 & D0 \\
\hline 0 & 0 & 0 & 1 & 0 & 1 & 1 & 0 \\
\hline
\end{tabular}

(47HC259)

Select IC21 (74HC259) Select Q6 in IC21

The input to XOR2 pin (13) is logic 0 . Therefore the XOR2 works as a buffer it will apply the high coming from the comp2 to INT2 pin (12) of the processor Z80180.

The output of the XOR1and XOR2 will depend on the two sides A, B of the two flow charts shown in Fig.8 and Fig.9. Table I describes the XOR1 output dependency.

Table I:

\begin{tabular}{|c||c||c||}
\hline $\begin{array}{c}\text { Comparator } \\
\text { output }\end{array}$ & Control input at pin (10) of XOR1 & Output of XOR1 gate \\
\hline \hline high & $\begin{array}{c}\text { Logic } 0 \text { (from initial setting) } \\
\text { (so it works as a buffer) }\end{array}$ & High and no interrupt \\
\hline \hline low & Logic 0 and it woks as a buffer & $\begin{array}{c}\text { Low and the processor } \\
\text { interrupted and start } \\
\text { executing side A }\end{array}$ \\
\hline \hline low & $\begin{array}{c}\text { Logic } 1 \text { at the of side A from the } \\
\text { subroutine so it works as an inverter }\end{array}$ & \begin{tabular}{c} 
High and no interruption \\
\hline \hline high
\end{tabular} \\
\hline \hline high & $\begin{array}{c}\text { Logic } 1 \text { and it works as an inverter } \\
\text { works as a buffer }\end{array}$ & $\begin{array}{c}\text { Low and the processor } \\
\text { interrupted and start } \\
\text { executing side B }\end{array}$ \\
\hline \hline
\end{tabular}


Table II describes the contents of $(9290 \mathrm{H})$ after each interrupt.

Table II:

\begin{tabular}{||c||c|c|}
\hline \hline $\begin{array}{c}\text { After INT2 side } \\
\text { A }\end{array}$ & Contents of $(9290 \mathrm{H})$ in Binary & Contents of $(9290 \mathrm{H})$ in Hex \\
\hline $\begin{array}{c}\text { After INT1 side } \\
\text { A }\end{array}$ & 00010001 & $11 \mathrm{H}$ \\
\hline $\begin{array}{c}\text { After INT1 side } \\
\text { B }\end{array}$ & 00110011 & $13 \mathrm{H}$ \\
\hline $\begin{array}{c}\text { After INT2 side } \\
\text { B }\end{array}$ & 00010011 & $03 \mathrm{H}$ \\
\hline
\end{tabular}

After this executing sequence for INT1 and INT2 subroutines, the flag byte stored in memory location $(9290 \mathrm{H})$ will contain $(03 \mathrm{H})$. Whenever the displaying program flips any required dot. It outs the data and the address required for flipping that dot. After a while it checks the flag byte stored in $(9290 \mathrm{H})$. if the displaying program detect that $03 \mid \mathrm{h}$ is stored in $9290 \mid \mathrm{h}$, it will continue displaying the other dots. If not, the timer programmable reload register will count down ten times (about $5 \mathrm{mSec}$ ) then the displaying program will switch OFF all transistors in the driver board, then it will continue displaying the other dots.

\section{Fault Detection}

From the previous section, it is clear that the flag byte stored in $(9290 \mathrm{H})$ will give an indication about the flipping process and the execution of the two subroutines due to the two interrupt signals $\overline{I N T 1}$ and $\overline{I N T 2}$. In the flipping circuit shown in Fig.1., supposing that T46 is not working (faulty transistor), examining the circuit in the following tow cases

Case 1: T46 is broken (open circuit)

a. If the transistor is open and we want to flip the dot in the direction caused by making T32 \& $\mathrm{T} 17$ switched $\mathrm{ON}$ and T46 \& T3 switched OFF. In that case we find that the broken transistor T46 has no effect and the dot will flip without any problem.

b. If we want to flip the dot to the other direction caused by making T46\&T3 switched ON and T32\&T17 switched OFF, we find that the dot will not flip and no current will pass through the resistor R22. Therefore $\overline{I N T 1}$ and $\overline{I N T 2}$ will not work, then flag byte stored in $(9290 \mathrm{H})$ will contain $(00 \mathrm{H})$.

Case 2:

a. If T46 is short and we want to flip the dot in the direction caused by making T32\&T17 OFF and T46\&T3 ON. In this case we find that the dot will flip normally. The flag byte stored in $(9290 \mathrm{H})$ contains $(03 \mathrm{H})$.

b. If T46 is short and we want to flip the dot in the direction caused by making T32\&T17 ON and T46\&T3 OFF. The 30V will transfer through the two diodes to the collector of T17, which will be switched ON causing a rectangular pulse at R22 as shown in Fig.12. No current will pass through the coil. Therefore no flipping occurred. The voltage drop across R22 is $8 \mathrm{~V}$ rectangular with $100 \mu \mathrm{Sec}$ duration causing the wave shaping circuit to charge until reaching $5.2 \mathrm{v}$ then discharging again to zero as shown in Fig.13. Comparing this new signal with $2 \mathrm{~V}$ and $3.4 \mathrm{v}$ in comp 1 and comp2 will cause the $\overline{I N T 1}$ and $\overline{I N T 2}$ to be as depicted in fig. 14 . it is obvious; there are differences between the $\overline{I N T 1}$ and $\overline{I N T 2}$ in the case of normal and faulty 
transistors. $\overline{I N T 1}$ Will broaden and overlap in time with $\overline{I N T 2}$ as depicted in the fig. 15 . In case of no short circuit the processor will execute INT2 subroutine, this will write $(11 \mathrm{H})$ in $(9290 \mathrm{H})$, and stay for about $25 \mu \mathrm{Sec}$ before executing INT1 subroutine as depicted in fig. 15 . In case of T46 is short circuit the processor will execute INT2 subroutine and because of the overlapping between the two interrupts it will directly execute INT1 subroutine. This will write $(33 \mathrm{H})$ in memory location $(9290 \mathrm{H})$. by checking this byte after the first return from interrupt, finding $(33 \mathrm{H})$ stored in it means that there is a short circuit transistor, the byte stored in $(9290 \mathrm{H})$ is checked twice, after first return from interrupt to check if there is a short circuit transistor and after about $500 \mu \mathrm{Sec}$ to check if there is a broken transistor.

Table III demonstrates the content of the memory location in $(9290 \mathrm{H})$ and its indications:

Table III:

\begin{tabular}{|l|l|}
\hline $\begin{array}{l}\text { Content } \\
\text { of } 9290 \mid \mathrm{h}\end{array}$ & Indication \\
\hline 03 & No fault \\
\hline 00 & Open circuit (broken transistor) \\
\hline 33 & Short circuit transistor \\
\hline
\end{tabular}

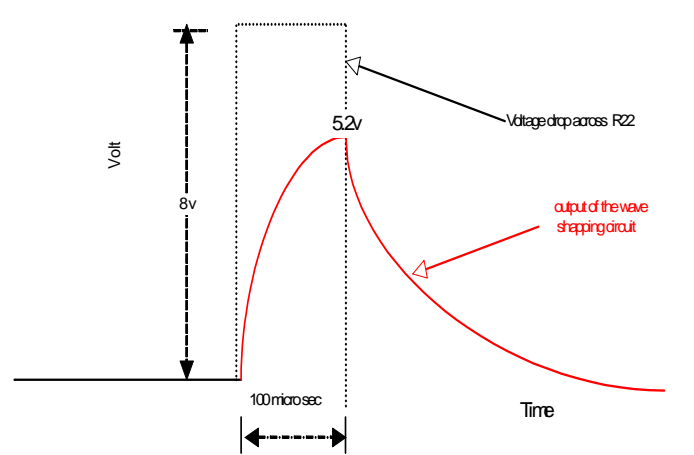

T46 Short

Fig.12. Voltage across R22 "point E and GND"

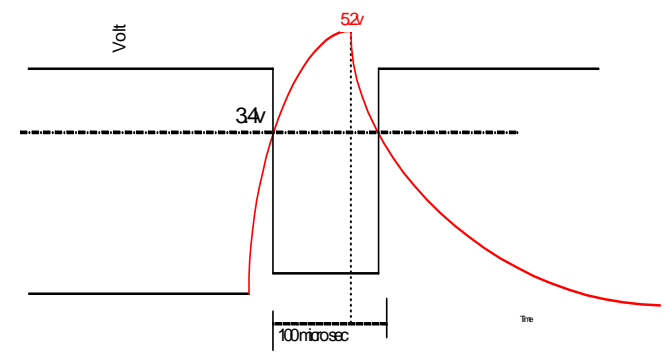

T46 Short

Fig.13. Pins "4","6" IC4 comparator

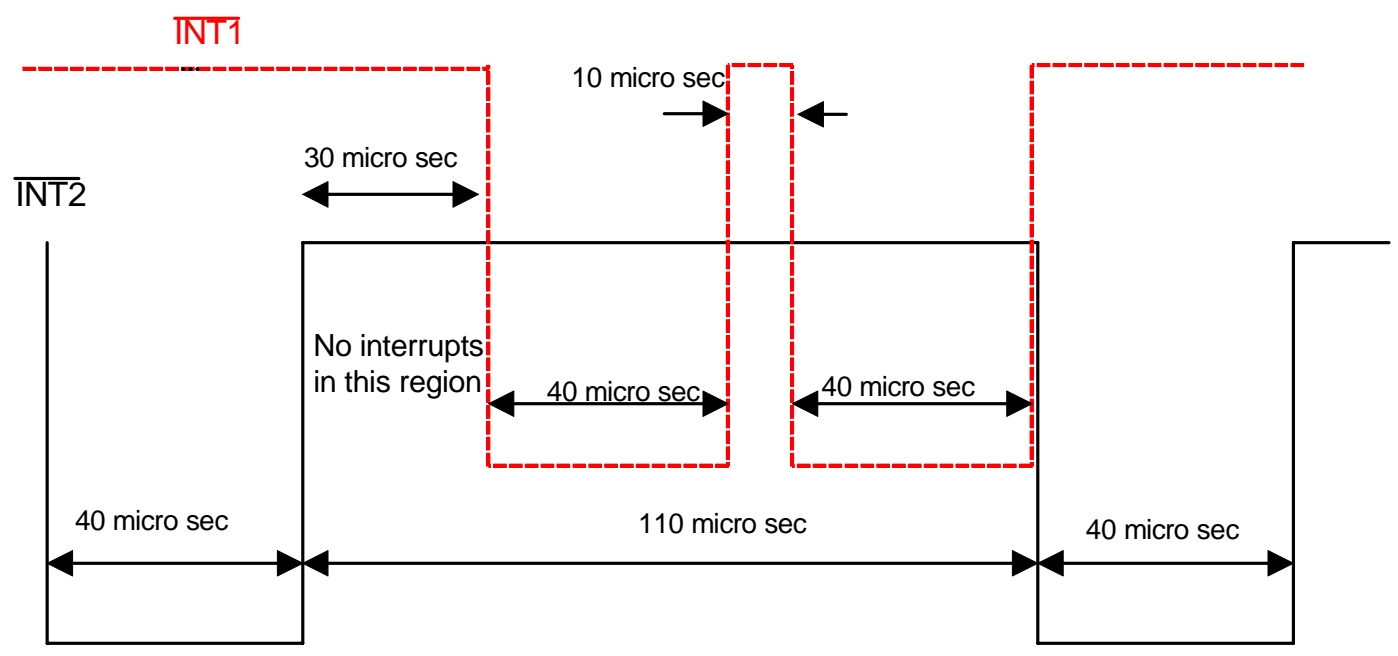

Fig.14. Timing diagram for INT1 and INT2 in case of No Fault 


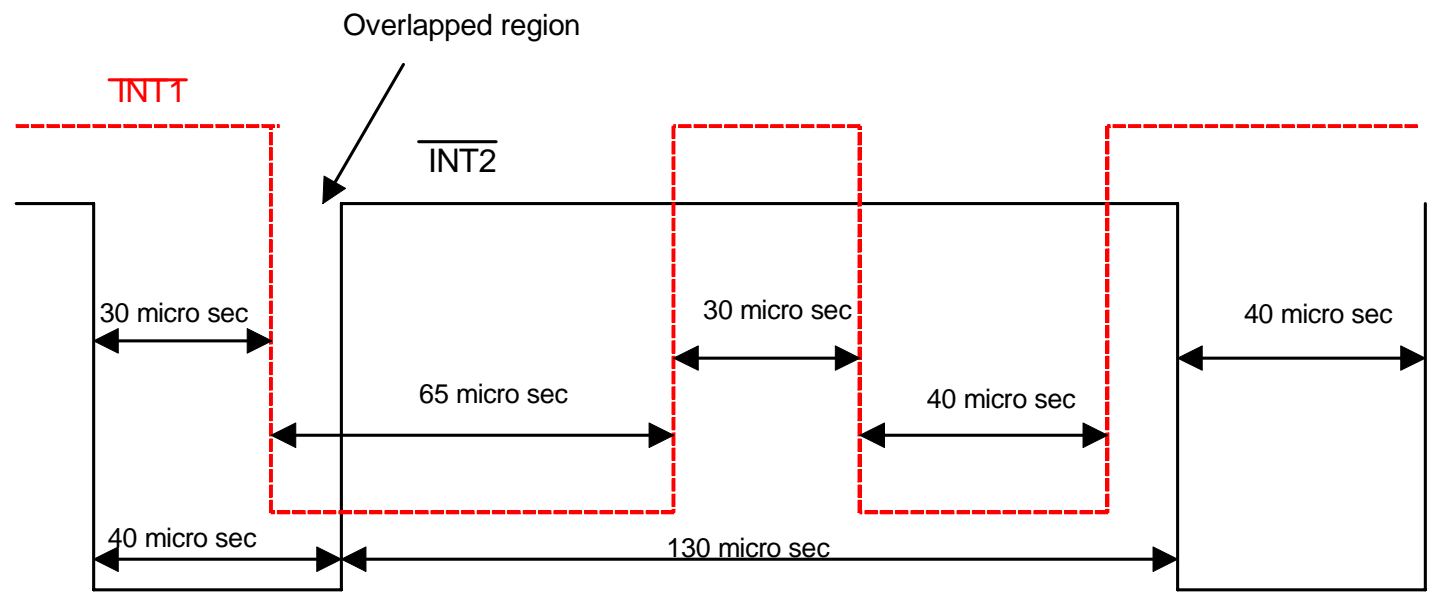

Fig.15. Timing diagram for INT1 and INT2 in case of Short Transistor

\section{Conclusion}

Fault detection for displayed data in signal state boards is very important. We have analyzed in details the schematic diagram of the SSB driving board. We have studied the existing PC and SSB software programs. We have extracted the hexadecimal program loaded in the driver board EPROM and disassembled to investigate it. We have performed a visual basic program to control the SSB instead of the old one written in Pascal to cope with the difficulties result in executing the Pascal program using high speed computers. The program includes a new added fault tracing section to detect the error in displaying and gives an alarm to the station operator to deal with this error by use another way to tell the right messages to the vessel.

\section{References}

[1] Ramesh S. Gaonkar, The Z80 microprocessor: Archtecture, Interfacing, Programming and Design, Prentice Hall, 1993.

[2] Paul Horowitz, Winfield Hill, The art of electronic, Cambridge University press 1983.

[3] C.J.Savant ,Martine S Roden,Gordon L. Carpenter, Electronic Design, The Benjamin / Cumming Publishing Company Inc.

[4] D.S.Yadav, Arun Kumar Singh, Microcontroller: Features and Applications, New AGE International (P), Publishers.

[5] R. W. Hanning, Coding and Information Theory, A prentice-Hall, 1980.

[6] Wolfgang W. Gartner, Transistors :priciples, Design and Applications, D. Van Nostrand Inc, 1980. 